Assessment of Rate of Force Development for Egyptian Handball National Team Using Wireless 3 Axis Aceelerometer.

\title{
Marwan Ragab
}

Department of Games Training, Faculty of Physical Education for Boys, Alexandria University, Egypt.

\section{Mansour Attaallah}

Department of Physical Education Foundation, Faculty of Physical Education for Boys, Alexandria University, Egypt.

\begin{abstract}
The purpose of this study was to examine the relationship between the rate of force development with two legs jump (2LEG) and one leg jump from three steps running (1LEG) for Egyptian handball national team players. Fifteen elite male handball players from the Egyptian handball national team (age=22.8 \pm 2.1 ; height=187.1 $\pm 7.2 \mathrm{~cm}$; mass=88.9 $\pm 10.1 \mathrm{~kg}$ ) volunteered in this study. Each player performed three trials of vertical jump (VJ) with two legs and three trials one leg jump from three steps running (1LEG). The best trial was selected for analysis. Wireless accelerometer data (Beanair AX-3DS 24G) were processed using Smoothing Moving Average filter (Sigview 2.5 software) to calibrate Peak of Force $(P F)$ and Time to Peak force (TPF) with a force platform data (Bertec). The results showed that rate of force development (RFD) has a relationship between the two legs jump (2LEG) and one leg jump from three steps running (1LEG) performance. The rate of force development (RFD) had a relationship with $2 L E G$ and $1 L E G$ performance. In high level handball, the rate of force development is the most important ability in most game situations; it gives the player an advantage to accelerate, decelerate or jump and shooting in less time. Therefore it can be used as an indicator for efficiency for all power activities and as a differential parameter between different classes of players in handball.
\end{abstract}

Keywords: Rate of force development, vertical jump, handball, accelerometer.

\section{Introduction:}

$\mathrm{H}$ andball can be characterized by high intensity actions performed at high velocities (Rogulj, Srhoj, \& Srhoj, 2004). This team sport, became much popular, in fact, Egyptian handball national team became the first non-European team to reach the World Championship semi-finals in the 2001 Men's tournament and its champion of the Mediterranean Games (2013). The vertical jump (VJ) with two legs jump (2LEG) is an important predictor of performance in various sports that require explosive actions such as in speed races, volleyball and handball which measures the effectiveness of training programs_(Hara, Shibayama, Takeshita, \& Fukashiro, 2006; Hennessy \& Kilty, 2001; Kraska et al., 2009; Peterson, Alvar, \& Rhea, 2006). The one leg jump from three steps running (1LEG) is used in handball shots to end the attack situations. The VJ height has been widely used by sports performance professionals as an alternative to direct assessment of maximal force and power (Arteaga, Dorado, Chavarren, \& Calbet, 2000; Hara et al., 2006)Recently, the validity of predicting Peak of Power (PP) and Average Power_(AP) on the basis of VJ height has been challenged (Carlock et al., 2004; Garhammer, 1993; G. J. Wilson, Murphy, \& Giorgi, 1996)._Previous studies showed the level of Peak of force (PF), the time to Peak force (TPF) and rate of force development (RFD) are related to performance in VJ (Cronin \& Sleivert, 2005; Haff et al., 1997; McLellan, Lovell, \& Gass, 2011; Stone et al., 2003; Vanezis \& Lees, 2005; Greg J. Wilson, Lyttle, Ostrowski, \& Murphy, 1995). Although the force platforms are widely used in the previous studies, they are expensive and not easily to accessible outside the laboratory settings. Nowadays the accelerometers have become small in size, very accurate, solid and less expensive (Kavanagh \& Menz, 2008; Keijsers, Horstink, \& Gielen, 2003; Moe-Nilssen \& Helbostad, 2002). Therefore, the main aim of the present study was examine the relationship between the rate of force development with two legs jump (2LEG) and one leg jump from three steps running (1LEG) using the accelerometer as an alternative measurement tool.

\section{Methods.}

Subject: Fifteen elite male handball players from the Egyptian handball national team $(\mathrm{age}=22.8 \pm 2.1 \mathrm{y}$; height $=187.1 \pm 7.2 \mathrm{~cm}$; mass $=88.9 \pm 10.1 \mathrm{~kg}$ ) volunteered in this study. They were also informed about protocol procedures. The protocol was constructed in accordance with the Declaration of Helsinki.

Procedures: Before collecting the data a Beanair_AX-3DS 24G wireless accelerometer was calibrated with a Bertec force platform. Data was processed using Smoothing 
moving average filter Sigview 2.5 software to calibrate Peak of Force (PF) and Time to Peak force (TPF)._To obtain the Peak of Force (PF) from accelerometer data (Peak of Ggravity unit (PG) $\times$ body weight) been calculated, and Time to Peak force (TPF) is same as the Time to Peak Force (TPF). Accelerometer was placed on the player's waist and was fixed with specific belt to avoid falling or moving. Each player perform three trials of two leg jump and three trials one leg jump from three steps running. The best trial was selected for analysis.

\section{Statistical analyses:}

Descriptive statistics, person correlation were used to examine the relationship between PF and TPF from force platform and accelerometer and, secondly, to evaluate the relationships between RFD to PF, TPF and height jump. The Bland Altman Plot method were used to measure agreement with repeated measures force platform and accelerometer. Significance was maintained at the 0.05 level IBM SPSS Statistics 21 software was used for the statistical analysis of the data.

\section{Results:}

The calibration result showed high significant correlation $(\mathrm{p}<0.01)$ between PF and TPF form force platform and accelerometer (Table 1). Bland Altman plots method showed that all values were confined between (SD \pm 1.96 ) which means that there is no significant differences between the two devices (as showed in Figure 1 and Figure 2). Table 2 shows the Mean \pm SD for biomechanics parameter rate of force development, peak of force and time to peak force. Tables 3 showed significant Correlation $(\mathrm{p}<0.05)$ between RDF and PF and significant Correlation $(\mathrm{p}<0.01)$ between RDF and TPF and there was no significant between RDF and VJD in 2LEG jump. There was a significant Correlation $(p<0.01)$ between RDF and PF, TPF while there was no significant between RDF and VJD in 1LEG jump.

Table 1:

Correlation between PF and TPF from force platform and accelerometer $\mathrm{n}=(7)$

\begin{tabular}{|c|c|}
\hline Parameters & $\mathrm{R}$ \\
\hline $\mathrm{PF}$ & $0.962^{* *}$ \\
\hline $\mathrm{TPF}$ & $0.985^{* *}$ \\
\hline
\end{tabular}

$* *$ Significant at $\mathrm{p}<0.01$

Figure 1:

bland-Altman Plot for between Peak of force from force platform and accelerometer.

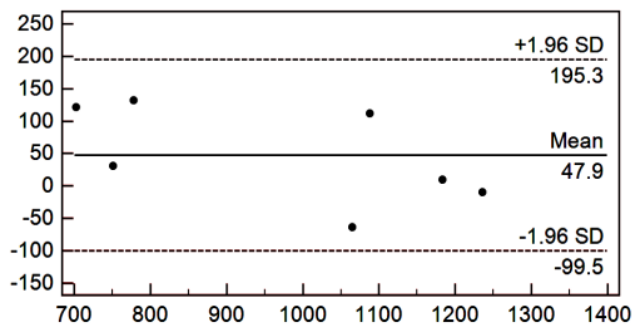

Figure 2:

bland-Altman Plot for between Time to Peak of force from force platform and accelerometer.

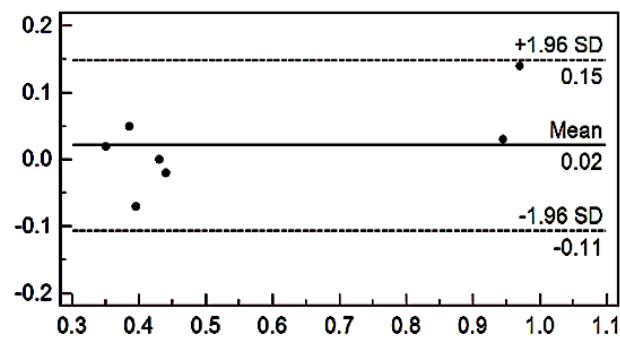

Table 2:

Descriptive values Mean \pm SD for average rate of force development, peak of force and time to peak force $(n=15)$

\begin{tabular}{|c|c|c|c|c|}
\hline Type of jump & \multicolumn{2}{|c|}{ Variables } & Mean & Std. Deviation \\
\hline \multirow{4}{*}{ 2LEG } & \multicolumn{2}{|c|}{ RFD( N.sec-1) } & 7268.70 & 2421.68 \\
\hline & \multicolumn{2}{|c|}{$\mathrm{PF}(\mathrm{N})$} & 1566.24 & 219.92 \\
\hline & TPF & $(\mathrm{S})$ & 0.237 & 0.078 \\
\hline & VJH & $(\mathrm{cm})$ & 0.47 & 0.05 \\
\hline \multirow{4}{*}{ 1LEG } & \multicolumn{2}{|c|}{ RFD2( N.sec-1) } & 16870.25 & 5600.42 \\
\hline & \multicolumn{2}{|c|}{$\mathrm{PF} 2(\mathrm{~N})$} & 2064.92 & 418.00 \\
\hline & TPF2 & $(\mathrm{S})$ & 0.130 & 0.031 \\
\hline & VJH2 & (cm) & 0.48 & 0.07 \\
\hline
\end{tabular}


Table 3:

Correlation between rate of force development, peak of force and time to peak force $(n=15)$

\begin{tabular}{|c|c|c|c|c|c|c|c|c|c|}
\hline \multirow{2}{*}{$\begin{array}{l}\text { Type of } \\
\text { jump }\end{array}$} & \multirow{2}{*}{ Parameters } & \multicolumn{4}{|c|}{ 2LEG } & \multicolumn{4}{|c|}{ 1LEG } \\
\hline & & RFD & $\mathrm{PF}$ & TPF & VJD & RFD & $\mathrm{PF}$ & TPF & VJD \\
\hline \multirow{4}{*}{ 2LEG } & RFD(N.s-1) & & & & & & & & \\
\hline & $\mathrm{PF} \quad(\mathrm{N})$ & $0.572 *$ & & & & & & & \\
\hline & TPF (s) & $-0.895 * *$ & -0.273 & & & & & & \\
\hline & VJD $(\mathrm{cm})$ & 0.223 & .0178 & -0.265 & & & & & \\
\hline \multirow{4}{*}{ 1LEG } & RFD_(N.s-1) & $0.574 *$ & 0.591* & -0.321 & -0.022 & & & & \\
\hline & $\mathrm{PF} \quad(\mathrm{N})$ & 0.492 & 0.491 & -0.278 & 0.052 & $0.911^{* *}$ & & & \\
\hline & TPF (s) & -0.391 & -0.390 & 0.218 & 0.248 & $-0.817 * *$ & $-0.568 *$ & & \\
\hline & VJD $(\mathrm{cm})$ & 0.383 & -0.310 & $-0.557 *$ & 0.363 & 0.049 & 0.066 & 0.045 & \\
\hline
\end{tabular}

*Significant at $\mathrm{p}<0.05$

**Significant at $\mathrm{p}<0.01$

$2 \mathrm{LEG}=$ two legs jump; $1 \mathrm{LEG}=$ one leg jump from three steps running; $\mathrm{RFD}=$ rate of force development two leg jump; $\mathrm{PF}=$ Peak of Force; $\mathrm{TPF}=$ time to Peak Force.

\section{Discussion:}

Previous studies have used height jump to assess the power from VJ (Aagaard, Simonsen, Andersen, Magnusson, \& Dyhre-Poulsen, 2002; Cronin \& Sleivert, 2005; Haff et al., 1997; Vanezis \& Lees, 2005; Greg J. Wilson et al., 1995). In the present study we examined the relationship between the RFD from two legs and one leg jump from three steps running for Egyptian handball national team. The RFD in VJ with two legs had a positive significant correlation with peak force $(r=0.572 \mathrm{p}<0.05)$, meaning that when the peak of force increase the RFD increase. The RFD had a negative significant correlation with time to peak force_( $r=-0.895 \mathrm{p}<0.01$ ) which mean when this decreased the RFD increase. In this result RFD had correlate to VJD ( $r=-0.383)$ and it is contrast with the results of other studies (Juliano Dal Pupo, 2011; Nuzzo, McBride, Cormie, \& McCaulley, 2008). The peak of force had no significant correlation to TPF $(r=-0.273)$ but the direction of this correlation is negative. According to this result we can predict that we cannot maintain to maximum Force for a long period of time. In handball this type of power is related with two important parameters Force and Velocity $\left(\mathrm{P}=\mathrm{F}^{*} \mathrm{~V}\right)$. In this case, the velocity is so important to apply the specific value of force (Challis, 1998), and is not necessary to be maximum force but the important how to apply this force within minimum time. The RFD in one leg jump had high positive significant correlation with peak of force $(r=0.911 \mathrm{p}<0.01)$, meaning that when peak of force increase the RFD increase and the RFD had a negative significant correlation to time to peak force $(r=-0.817 \mathrm{p}<0.01)$, meaning that when this time decreased the RFD increase. The peak of force had negative significant correlation to TPF_(r=-0.568 $p<0.01)$ also RFD had poor negative correlate to VJD. ( $\mathrm{r}=-0.049)$. There was a positive significant correlation between RFD in two legs jump and RFD in one leg jump from three steps $(r=0.574 \mathrm{p}<0.05)$. Although the correlation values are not significant between RFD in two legs jump and peak of force, time to peak force in one leg jump the direction of correlation are similar with peak of force, time to peak force in two legs jump. There are two type of jumps examine the player's ability to develop force rapidly during static potion (two leg jump) and dynamic potion (one leg jump from three steps running). This explains the great different between the mean of RFD in the two legs jump (7268.70 \pm 2421.68$)$ and the one leg jump from three steps running $(16870.25 \pm 5600.42)$. That is because the body had accelerated before jump. Therefore it has been found that the time to peak force $(0.130 \pm 0.031)$ in one leg jump from three steps running less than the time to peak force $(0.237 \pm 0.078)$ in two leg jump this agreed with (Row, 1999). In this time the player try to overcome his inertia and gravity. The mean VJD from two legs is $(0.47 \pm 0.05)$ and the mean VJD from one leg is $(0.48 \pm$ 0.07). the one-leg and two-legs vertical jumps do not different in jump height in a group of athletes accustomed to their use(ow, 1999). the study of (Peter F. Vint, 1996) quantified the differences between one-leg and two-legs vertical jumping performances and they reported greater flight heights during two-foot jumps, as expected, but the magnitude of this difference was only about $9 \mathrm{~cm}$. this study using a running approach for one and two legs jump . The one leg jumps might benefit from an increased take off height which is largely attributable to horizontal approach velocity. The VJ from running in handball may take the shape up and forward direction so the net force is higher with two directions. the RFD play a great role for both type of jumps countermovement jump with two legs and one leg jump from three steps running as most 
common performance used in the power activity. (Arteaga et al., 2000; Stone et al., 2003).

\section{Conclusion:}

The results of this study showed that rate of force development had a relationship with two legs and one leg performance. In high level handball, the rate of force development is the most important ability in most game situations; it gives the player an advantage to accelerate, decelerate or jump and shooting in less time. Therefore it can be used as an indicator for efficiency for all power activities and as a differential parameter between different classes of players in handball.

\section{References:}

1. Aagaard, P., Simonsen, E. B., Andersen, J. L., Magnusson, P., \& Dyhre-Poulsen, P. (2002). Increased rate of force development and neural drive of human skeletal muscle following resistance training. J Appl Physiol (1985), 93(4), 1318-1326. doi: 10.1152/japplphysiol.00283.2002

2. Arteaga, R., Dorado, C., Chavarren, J., \& Calbet, J. A. (2000). Reliability of jumping performance in active men and women under different stretch loading conditions. J Sports Med Phys Fitness, 40(1), 26-34.

3. Carlock, J. M., Smith, S. L., Hartman, M. J., Morris, R. T., Ciroslan, D. A., Pierce, K. C., . . . Stone, M. H. (2004). The relationship between vertical jump power estimates and weightlifting ability: a fieldtest approach. J Strength Cond Res, 18(3), 534-539. doi: $10.1519 / \mathrm{r}-13213.1$

4. Challis, J. H. (1998). An investigation of the influence of bi-lateral deficit on human jumping. Human Movement Science 17, 307-325.

5. Cronin, J., \& Sleivert, G. (2005). Challenges in understanding the influence of maximal power training on improving athletic performance. Sports Med, 35(3), 213-234.

6. Garhammer, J. (1993). A Review of Power Output Studies of Olympic and Powerlifting: Methodology, Performance Prediction, and Evaluation Tests. The Journal of Strength \& Conditioning Research, 7(2), 76-89.

7. Haff, G. G., Stone, M., O'Bryant, H. S., Harman, E., Dinan, C., Johnson, R., \& Han, K.-H. (1997). ForceTime Dependent Characteristics of Dynamic and Isometric Muscle Actions. The Journal of Strength \& Conditioning Research, 11(4), 269-272.
8. Hara, M., Shibayama, A., Takeshita, D., \& Fukashiro, S. (2006). The effect of arm swing on lower extremities in vertical jumping. J Biomech, 39(13), 2503-2511. doi: 10.1016/j.jbiomech.2005.07.030

9. Hennessy, L., \& Kilty, J. (2001). Relationship of the stretch-shortening cycle to sprint performance in trained female athletes. J Strength Cond Res, 15(3), 326-331.

10. Juliano Dal Pupo, D. D. a. S. G. d. S. (2011). Kinetic Parameters Determinants of Performance in Counter Movement Jump. Portuguese Journal of Sport Sciences, 11(2).

11. Kavanagh, J. J., \& Menz, H. B. (2008). Accelerometry: a technique for quantifying movement patterns during walking. Gait Posture, 28(1), 1-15. doi: 10.1016/j.gaitpost.2007.10.010

12. Keijsers, N. L., Horstink, M. W., \& Gielen, S. C. (2003). Online monitoring of dyskinesia in patients with Parkinson's disease. IEEE Eng Med Biol Mag, 22(3), 96-103.

13. Kraska, J. M., Ramsey, M. W., Haff, G. G., Fethke, N., Sands, W. A., Stone, M. E., \& Stone, M. H. (2009). Relationship between strength characteristics and unweighted and weighted vertical jump height. Int J Sports Physiol Perform, 4(4), 461-473.

14. McLellan, C. P., Lovell, D. I., \& Gass, G. C. (2011). The role of rate of force development on vertical jump performance. J Strength Cond Res, 25(2), 379385. doi: 10.1519/JSC.0b013e3181be305c

15. Moe-Nilssen, R., \& Helbostad, J. L. (2002). Trunk accelerometry as a measure of balance control during quiet standing. Gait Posture, 16(1), 60-68.

16. Nuzzo, J. L., McBride, J. M., Cormie, P., \& McCaulley, G. O. (2008). Relationship between countermovement jump performance and multijoint isometric and dynamic tests of strength. J Strength Cond Res, 22(3), 699-707. doi: 10.1519/JSC.0b013e31816d5eda

17. Peter F. Vint, R. N. H. (1996). Differences Between One-Foot and Two-Foot Vertical Jump Performances. JAB, 12(3), 338 - 358 .

18. Peterson, M. D., Alvar, B. A., \& Rhea, M. R. (2006). The contribution of maximal force production to explosive movement among young 
collegiate athletes. J Strength Cond Res, 20(4), 867873. doi: $10.1519 / \mathrm{r}-18695.1$

19. Rogulj, N., Srhoj, V., \& Srhoj, L. (2004). The contribution of collective attack tactics in differentiating handball score efficiency. Coll Antropol, 28(2), 739-746.

20. Row, B. S. (1999). The one-legged and two-legged vertical jumps a temporal, kinetic and electromyographic analysis. Available from http://worldcat.org /z-wcorg/ database.

21. Stone, M. H., O'Bryant, H. S., McCoy, L., Coglianese, R., Lehmkuhl, M., \& Schilling, B. (2003). Power and maximum strength relationships during performance of dynamic and static weighted jumps. J Strength Cond Res, 17(1), 140-147.
22. Vanezis, A., \& Lees, A. (2005). A biomechanical analysis of good and poor performers of the vertical jump. Ergonomics, 48(11-14), 1594-1603. doi: 10.1080/00140130500101262

23. Wilson, G. J., Lyttle, A. D., Ostrowski, K. J., \& Murphy, A. J. (1995). Assessing Dynamic Performance: A Comparison of Rate of Force Development Tests. The Journal of Strength \& Conditioning Research, 9(3), 176-181.

24. Wilson, G. J., Murphy, A. J., \& Giorgi, A. (1996). Weight and plyometric training: effects on eccentric and concentric force production. Can J Appl Physiol, 21(4), 301-315. 
